3 - ORIGINAL ARTICLE

ISCHEMIA-REPERFUSION

\title{
Remote limb ischemic post-conditioning attenuates ischemia-reperfusion injury in rat skin flapby limiting oxidative stress ${ }^{1}$
}

\author{
Yi ZhangI, Hua Xu' ${ }^{I I}$, Tao Wang ${ }^{I I I}$, Jinguang $\mathrm{He}^{\mathrm{IV}}$, Jiao Wei ${ }^{\mathrm{IV}}$, Tingliang Wang ${ }^{\mathrm{IV}}$, Jiasheng Dong ${ }^{\mathrm{V}}$ \\ DOI: http://dx.doi.org/10.1590/S0102-865020160010000003
}

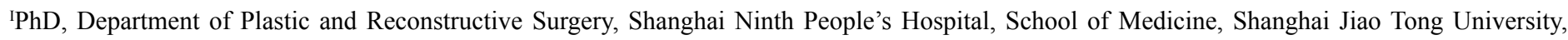
China. Acquisition and analysis of data, manuscript writing.

IIPhD, Assistant Professor, Department of Plastic and Reconstructive Surgery, Shanghai Ninth People's Hospital, School of Medicine, Shanghai Jiao Tong University, China. Design of the study, manuscript writing.

IIIPhD, Assistant Professor, Department of Plastic and Reconstructive Surgery, Shanghai Ninth People's Hospital, School of Medicine, Shanghai Jiao Tong University, China. Acquisition of data, carrying out the study.

${ }^{\text {IV }} \mathrm{PhD}$, Department of Plastic and Reconstructive Surgery, Shanghai Ninth People's Hospital, School of Medicine, Shanghai Jiao Tong University, China. Acquisition of data, carrying out the study.

vPhD, Full Professor, Department of Plastic and Reconstructive Surgery, Shanghai Ninth People's Hospital, School of Medicine, Shanghai Jiao Tong University, China. Design and supervised all phases of the study, critical revision.

\section{ABSTRACT}

PURPOSE: To investigate the effect of remote ischemic post-conditioning (RIPoC) against ischemia-reperfusion (I/R) injury on flaps of rats.

METHODS: Sprague-Dawley rats were randomized into the Sham, Control, RIPoC1 and RIPoC2 groups. All the animals were submitted to a $5 \times 4 \mathrm{~cm}$ superficial inferior epigastric artery flap. Eight hours of flap ischemia was induced and two protocols of limb RIPoC were applied. Tissue MDA level and SOD activity in 24-h reperfusion were assessed. Flap survival was assessed 7 days postoperatively.

RESULTS: Compared to the Control group, the RIPoC1 group showed statistically decreased MDA level at 6-, 12-, and 24-h reperfusion $(\mathrm{P}=0.01, \mathrm{P}<0.01$ and $\mathrm{P}<0.01$, respectively), and statistically increased SOD activity at 12 - and 24-h reperfusion $(\mathrm{P}<0.05$ and $\mathrm{P}<$ 0.01 , respectively). Flap survival rate on the 7th day was significantly higher in the RIPoC1 group than the control group (47.9 6.4 vs. $29.4 \pm 7.1 \%, \mathrm{P}<0.01)$.

CONCLUSION: Three cycles of 5-min Limb remote ischemic post-conditioning rather than a single cycle of 15 -min limb RIPoC has protective effect on flaps against ischemia-reperfusion injury by attenuating oxidative stress.

Key words: Ischemic Postconditioning. Ischemia. Reperfusion Injury. Oxidative Stress. Rats. 


\section{Introduction}

Free flap transplantation is frequently used in plastic and reconstructive surgery. In spite of the high success rate, reperfusion injury by abrupt restoration of circulation after prolonged ischemia has been remained an unsolved problem associated with partial to total flap loss.

Excessive production of free radicals and/or reactive oxygen species during reperfusion triggers lipid peroxidation and initiates ischemia reperfusion ( $/ / R)$ damage ${ }^{1}$. Malondialdehyde (MDA) is one of intermediate products of lipid peroxidation, and its level is commonly used as a biomarker of oxidative stress ${ }^{2}$. On the contrary, endogenous antioxidant enzymes, such as superoxide dismutase (SOD) and catalase, act as "free radical scavengers" which can transform superoxide anion to the less reactive species $\mathrm{H}_{2} \mathrm{O}_{2}{ }^{3}$.

In recent years, numerous labs have demonstrated that ischemic post-conditioning (IPostC), defined as brief intermittent episodes of ischemia at the onset of reperfusion, is an endogenous protective strategy against I/R injury for multiple organs ${ }^{4-6}$. The underling mechanism of IPostC involves limiting the oxidative stress by reducing reactive oxygen metabolites and increasing antioxidant enzymes' activity in tissues after prolonged ischemic insult $^{7}$. However, repetitive ischemic insult and hypoxia added directly on the primary ischemic tissue are still unacceptable concept and impracticable. Remote ischemic post-conditioning (RIPoC) refers to ischemic tolerance induced by transient ischemia at a site distant to the ischemic target organs immediately at the time of its reperfusion ${ }^{8}$. In contrast to the clinical limitation of IPostC, RIPoC could be a non-invasive clinical intervention. Recent preclinical papers show that RIPoC performed in the limbs can generate effective cardioprotection and neuroprotection against $\mathrm{I} / \mathrm{R}$ injury similar to IPostC $\mathrm{C}^{9,10}$, yet its protective effect on skin flap has not yet been well investigated.

In the present study, we aimed to evaluate the antiischemic properties of two protocols of limb RIPoC in a rat model of epigastric island flaps subjected to $\mathrm{I} / \mathrm{R}$ injury, and the possible effect of RIPoC on reduction of I/R-induced oxidative stress.

\section{Methods}

All procedures were conducted in compliance with NIH Guiding Principles for Research Involving Animals and were approved by the Institutional Animal Care and Use Committee of
Shanghai Jiao Tong University School of Medicine.

Male Sprague-Dawley (SD) rats, weighing between $290 \mathrm{~g}$ to $350 \mathrm{~g}$, were randomized into4four groups ( $\mathrm{n}=16$ in each group): the Sham, Control, RIPoC1 and RIPoC2 groups. The animals were kept in a room with a controlled environment with a 12-h day/ night lighting cycle, and allowed access to standard rodent chow and tap water. General anesthesia was induced with intraperitoneal injections of ketamine $80 \mathrm{mg} / \mathrm{kg}$ and maintained with a booster injection of one third of initial dose.

\section{Surgicaleprocedure}

The abdomen was shaved with an electric hair clipper, and asepsis was maintained by providing a local sterile. A $5 \times 4$ $\mathrm{cm}$ island pedicle flap was elevated in all animals with the base at left superficial inferior epigastric (SIE) vessels (Figure 1). The flap consisted of skin, subcutaneous tissue, and intimately attached panniculus carnosus. The SIE pedicle was carefully isolated down to the left femoral vessels, and de-nervated under a microscope to accurately mimic a free tissue transfer.

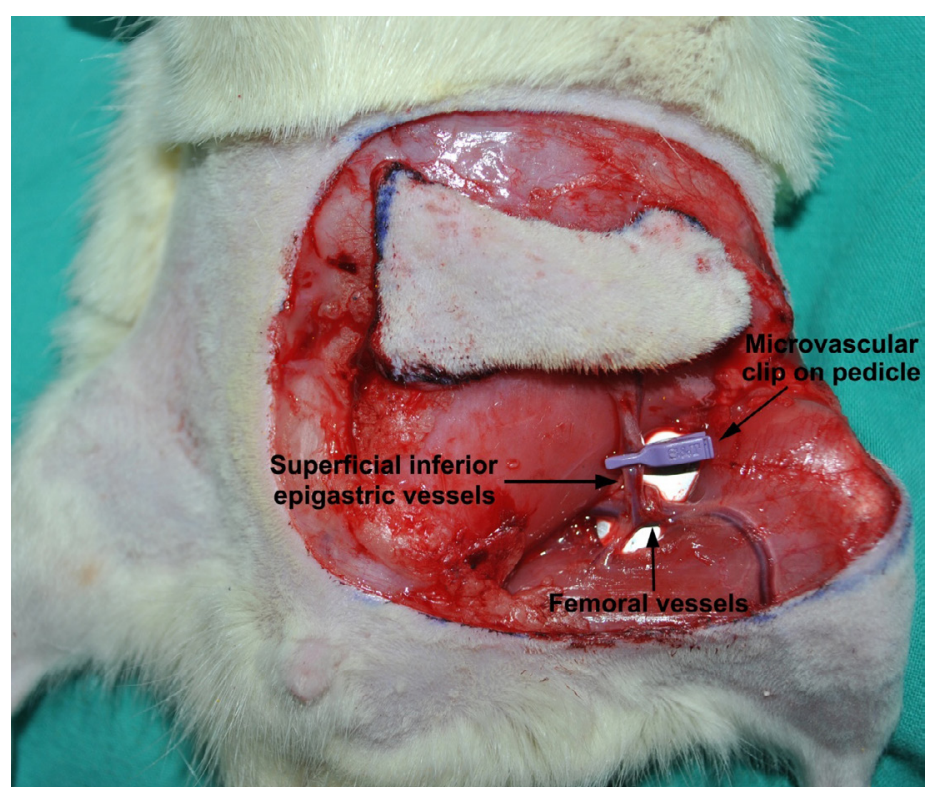

FIGURE 1 - An inferior epigastric flap $(5 \times 4 \mathrm{~cm})$ as a skin flap reperfusion injury model. The left superficial inferior epigastric pedicle (horizontal arrow) was carefully isolated down to the femoral vessels (vertical arrow). Flap ischemia was induced using an S\&T microvascular clip Ooblique arrow) with $15 \mathrm{~g}$ compression on the flap pedicle.

In the Control group, an 8-h flap ischemia was induced using an S\&T microvascular clip with $15 \mathrm{~g}$ compression on the pedicle. During the period of ischemia, the rats were kept 
anesthetized and the flap was placed in gauze moistened with warm saline solution. After the clip was removed, the vascular patency of the pedicles was confirmed under an operating microscope before the flap was repositioned and sutured. The Sham group underwent the same flap elevation and vessel dissection as the Control group, but flap I/R was not induced (Figure 2).

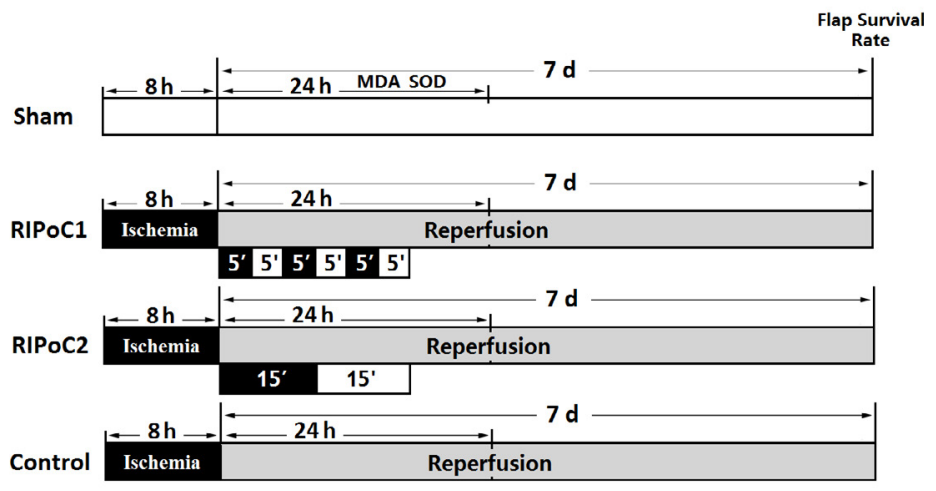

FIGURE 2 - Experimental protocol. Rats were randomized into 4 groups ( $\mathrm{n}=16$ in each group): (1) In the Sham group, rats underwent $8 \mathrm{hrs}$ of general anesthesia (open bar) and a sham operation; (2) In the Control group, rats underwent $8 \mathrm{hrs}$ of flap ischemia (dark bar) followed by reperfusion (gray bar) with no conditioning therapy; In the RIPoC groups, limb RIPOC was administered at the onset of flap reperfusion after $8 \mathrm{hrs}$ of ischemia. (3) RIPoC1 was achieved by 3 cycles of 5-min limb ischemia (dark bar) followed by 5-min reperfusion (open bar); (3) RIPoC2 was achieved by 1 cycles of 15 -min limb ischemia (dark bar) followed by reperfusion (open bar). Tissue MDA content and SOD activity within 24 hours after reperfusion was examined, and the flap survival area was evaluated at the $7^{\text {th }}$ day.

The RIPoC groups underwent the same procedure as the Control group, and 3 cycles of 5-min limb ischemia followed by 5-min reperfusion (RIPoC1), or 1 cycle of 15-min limb ischemia followed by limb reperfusion (RIPoC2), at the onset of flap reperfusion. Limb ischemia was induced by placing a tourniquet around the base of the right hind limb to stop the arterial blood supply. Circulatory arrest in the limbs was confirmed by observing the empurpled limb skin and via vascular Doppler (Philips). The rationale for the timing used in this experimental design was based on our experience and the literatures. All the animals were sacrificed by a lethal dose $(100 \mathrm{mg} / \mathrm{kg})$ of intracardiac Nembutal, after the observations were completed (Figure 3).
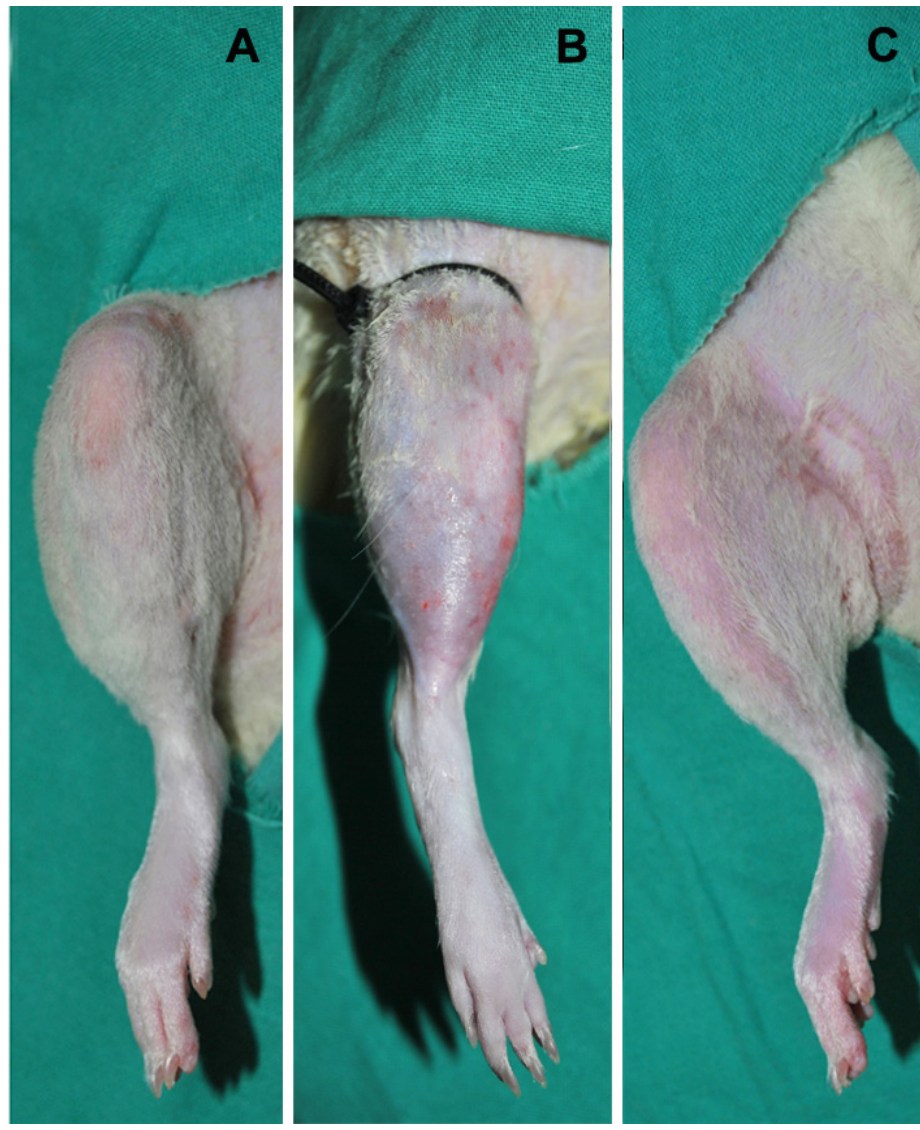

FIGURE 3 - Remote ischemic post-conditioning induced by placing a tourniquet around the base of the right hind limb. A. Normal limb; B. Ischemic limb; C. Reperfused limb.

\section{SOD activity and MDAtcontent}

A full-thickness, 2-mm punch biopsy of the flap skin wasecollected at the distal end of the flap, after $3 \mathrm{~h}, 6 \mathrm{~h}, 12 \mathrm{~h}, 24 \mathrm{~h}$ after reperfusion, respectively. The samples were rinsed, weighed, and homogenized in 9 volumes of ice-cold buffers. Supernatant homogenate was collected after centrifugation at $5000 \mathrm{rpm} / \mathrm{min}$ for $15 \mathrm{~min}$ at $4 \mathrm{C}$. The SOD activity of homogenates was determined by the xanthine oxidase (hydroxylamine) method, using a commercial kit (A001; Nanjing jiancheng Bioengineering Institute, China), and the results are expressed as $\mathrm{nmol} / \mathrm{mg}$ wet weight. The MDA content of homogenates was determined spectrophotometrically by thiobarbituric acid colorimetric method, using a commercial kit (A003; Nanjing jiancheng Bioengineering Institute, China), and the results are expressed as $\mu \mathrm{mol} / \mathrm{mg}$ wet weight. 


\section{Flap survival analysis}

At the $7^{\text {th }}$ day after reperfusion, the animal was reanesthetized and the appearance of the skin flap was recorded and photographed in a standardized fashion. The flap viable area was determined based on color, capillary refill, and the pin-prick test Ratios of viable area to original flap area were calculated by digital planimetry software (Image-Pro Plus Version 7.0), expressed as a percentage (percent survival).

\section{Statisticalsanalysis}

The results were expressed as mean + standard deviation (SD). Student t-test was used for comparisons between two means.
All data were analyzed using SPSS software 19.0. Values $<0.05$ were considered to be statistically significant.

\section{Results}

\section{MDA content and SOD activity}

Ischemia and the subsequent reperfusion resulted in significantly higher level of MDA content and lower level of SOD activity in flap tissue from both the Control and RIPoC groups than that in tissue from the Sham group, throughout the 24-h reperfusion period (Figure 4).

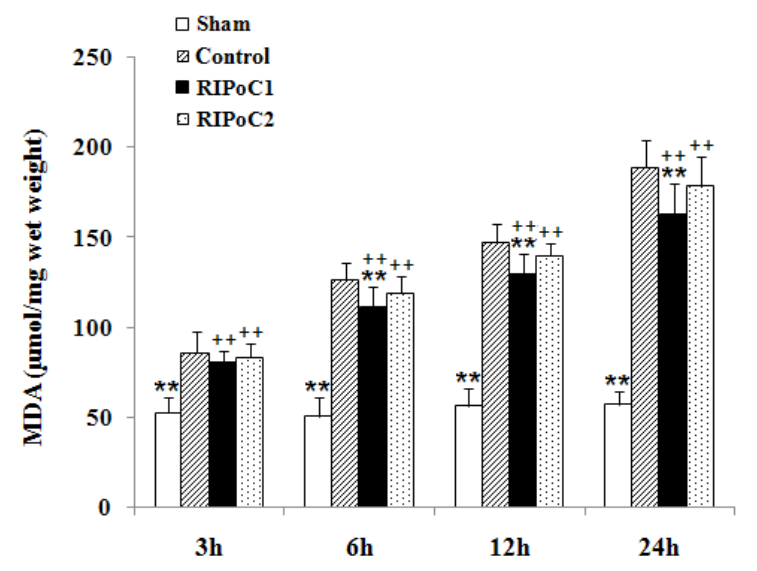

(A)

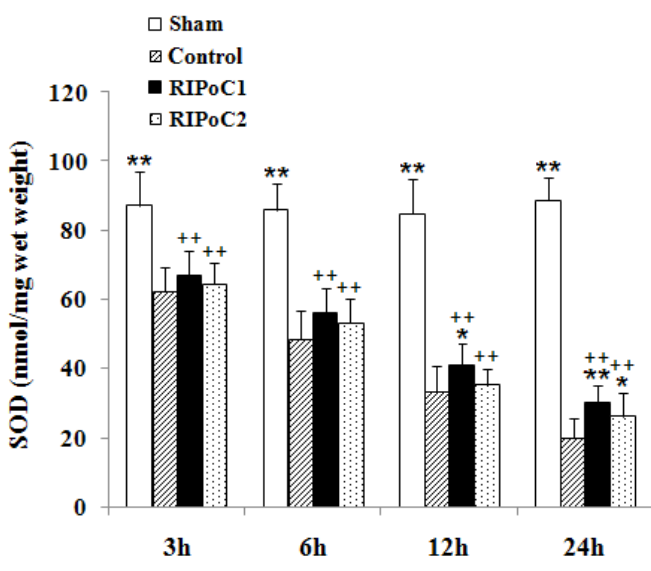

(B)

FIGURE 4 - Flap tissue content of malondialdehyde (MDA) (A) and activity of Superoxide dismutase (SOD) (B) at 3-, 6-, 12- and 24-h reperfusion in the Sham, Control and RIPoC groups ( $\mathrm{n}=8$ per group at each timepoint). ${ }^{*}: \mathrm{P}<0.05 v s$. Control group; ${ }^{* *}: \mathrm{P}<0.01 v s$. Control group; ${ }^{+}$: $<<0.05$ vs. Sham group; ${ }^{++}: \mathrm{P}<0.01$ vs. Sham group.

Compared with the controls, induction of $\mathrm{RIPoC} 1$ significantly attenuated I/R-induced elevation in MDA at 6-, 12-, and 24-h reperfusion timepoints $(\mathrm{P}=0.01, \mathrm{P}<0.01$ and $\mathrm{P}<0.01$, respectively). In contrast, differences in MDA level between the Control and RIPoC2 groups at each timepoint were not significant $(\mathrm{P}>0.05)$. Compared with the controls, RIPoC1 significantly attenuated the decrease in SOD activity at 12- and 24-h reperfusion timepoints ( $\mathrm{P}<0.05$ and $\mathrm{P}<0.01$, respectively) while RIPoC2 only attenuated the decrease in SOD activity at 24-h reperfusion timepoints $(\mathrm{P}<0.05)$. Detailed statistical evaluation of the MDA levels and SOD activity Tables 1 and 2).

TABLE 1 - MDA levels at each timepoint in each group.

\begin{tabular}{ccccccccc}
\hline Groups & Sham & \multicolumn{2}{c}{$\begin{array}{c}\text { Control } \\
\text { Avr. } \pm \text { SD }(\mu \mathrm{mol} / \mathrm{mg} \text { wet weight })\end{array}$} & RIPoC2 & P1 & P2 & P3 \\
\hline 3. h & $52.4 \pm 8.6$ & $86.1 \pm 11.2$ & $80.3 \pm 6.9$ & $83.1 \pm 7.5$ & $<0.01$ & 0.23 & 0.54 \\
6. h & $50.4 \pm 10.7$ & $126.6 \pm 9.0$ & $111.5 \pm 11.2$ & $119.1 \pm 9.6$ & $<0.01$ & 0.01 & 0.17 \\
12. h & $56.3 \pm 9.5$ & $147.2 \pm 10.0$ & $129.7 \pm 11.5$ & $139.8 \pm 6.6$ & $<0.01$ & $<0.01$ & 0.1 \\
24. h & $57.0 \pm 7.2$ & $188.7 \pm 15.6$ & $162.8 \pm 17.2$ & $179.0 \pm 15.8$ & $<0.01$ & $<0.01$ & 0.1 \\
\hline
\end{tabular}

$\mathrm{P}_{1}$ : Control group vs. Sham group; $\mathrm{P}_{2}$ : Control group vs. RIPoC1 group; $\mathrm{P}_{3}$ : Control group vs. RIPoC2 group; $\mathrm{p}$ value in bold when less than 0.05 . 
TABLE 2 - SOD levels at each timepoint in each group.

\begin{tabular}{ccccccccc}
\hline Groups & Sham & $\begin{array}{c}\text { Control } \\
\text { Avr. } \pm \text { SD (nmol/mg wet weight) }\end{array}$ & RIPoC2 & P1 & P2 & P3 \\
\hline 3. h & $87.0 \pm 10.1$ & $62.3 \pm 6.9$ & $66.7 \pm 7.5$ & $64.6 \pm 6.2$ & $<0.01$ & 0.24 & 0.48 \\
6. h & $85.8 \pm 7.7$ & $48.5 \pm 8.3$ & $56.3 \pm 6.9$ & $53.3 \pm 7.1$ & $<0.01$ & 0.06 & 0.23 \\
12.h & $84.8 \pm 9.9$ & $33.2 \pm 7.6$ & $41.0 \pm 6.5$ & $35.2 \pm 4.2$ & $<0.01$ & 0.05 & 0.54 \\
24. h & $88.6 \pm 6.8$ & $20.0 \pm 5.8$ & $30.2 \pm 4.9$ & $26.1 \pm 5.3$ & $<0.01$ & $<0.01$ & 0.04 \\
\hline
\end{tabular}

$\mathrm{P}_{1}$ : Control group $v s$. Sham group; $\mathrm{P}_{2}$ : Control group $v s$. RIPoC1 group; P3: Control group vs. RIPoC2 group; $\mathrm{p}$

\section{Flap survival rate}

The viable region of the flap was measured 7 days after reperfusion (Figure 5). Compared to the Sham group, significant decrease in ratios of the survival area to original flap area was observed in the Control group $(29.4 \pm 7.1$ vs. $92.0 \pm 4.4 \%$,
$\mathrm{P}<0.01)$, RIPoC1 group (47.9 \pm 6.4 vs. $92.0 \pm 4.4 \%, \mathrm{P}<0.01)$, and RIPoC2 group (34.4 44.2 vs. $92.0 \pm 4.4 \%, \mathrm{P}<0.01)$. Compared to the controls, induction of limb RIPoC1 significantly increased the flap survival rate $(\mathrm{P}<0.01)$. In contrast, difference in flap survival rate between the Control and RIPoC2 groups was not significant $(\mathrm{P}>0.05)$ (Table 3).
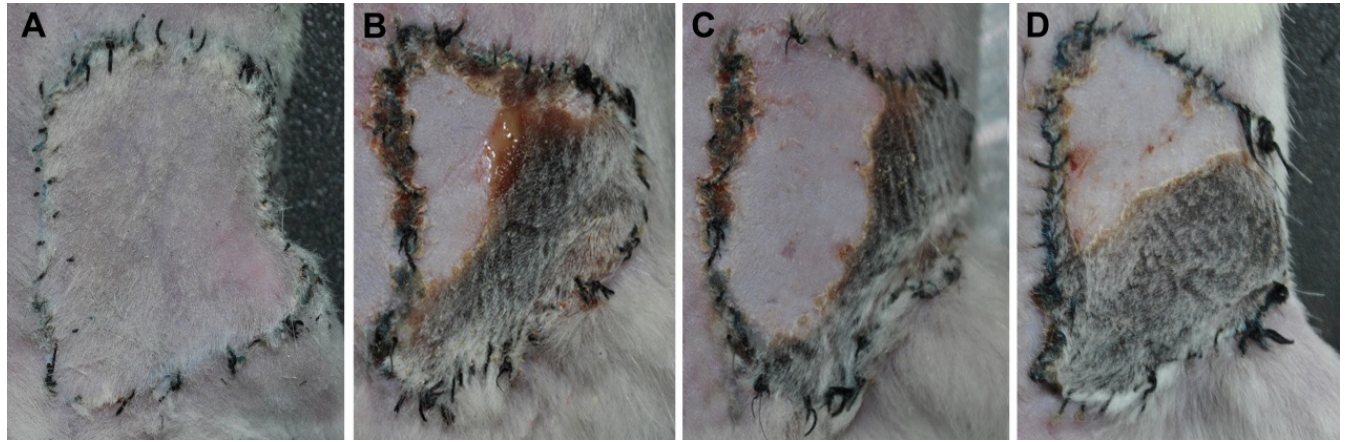

FIGURE 5 - Photographs of the typical flap of each group taken on the day 7 after reperfusion. The mean flap survival rate was $92.0 \pm 4.4 \%$ in Sham group (A), $29.4 \pm 7.1 \%$ in Control group (B), and $47.9 \pm 6.4 \%$ in RIPoC1 group (C), $34.4 \pm 4.2 \%$ in RIPoC2 group (D), respectively.

TABLE 3 - Flap survival rates in each group at the 7th day after reperfusion.

\begin{tabular}{cccccccc}
\hline Groups & Sham & Control & RIPoC1 & RIPoC2 & $\mathrm{P}_{1}$ & $\mathrm{P}_{2}$ & $\mathrm{P}_{2}$ \\
& & Avr. \pm SD $(\%)$ & & \\
\hline Survival rate & $92.0 \pm 4.4$ & $29.4 \pm 7.1$ & $47.9 \pm 6.4$ & $34.4 \pm 4.2$ & $<\mathbf{0 . 0 1}$ & $<\mathbf{0 . 0 1}$ & 0.11 \\
\hline
\end{tabular}

$\mathrm{P}_{1}$ : Control group vs. Sham group; $\mathrm{P}_{2}$ : Control group vs. RIPoC1 group; $\mathrm{P}_{3}$ : Control group vs. RIPoC2 group; $\mathrm{p}$ value in bold when less than 0.05.

\section{Discussion}

IPostC has been proven to confer protection against flap I/R injury. Moon et al. ${ }^{11}$ reported that IPostC could decrease flap necrosis by reducing MPO activity and attenuating acute inflammatory reaction. Experimental data from Coskunfirat et al. ${ }^{12}$ showed that IPostC applied by means of 6 cycles of 30 seconds yields an optimal anti-ischemia protection in a rat skin flap model.
In the present study, we expand further on this concept by applying post-conditioning at a distance (hind limbs) in a rat skin flap model, and showed that RIPoC was an effective therapeutic intervention against flap I/R injury.

The protective effect of RIPoC was initially described in the heart ${ }^{13}$, and applied to other organs ${ }^{14-16}$. RIPoC conducted in the limbs is most commonly used in the previous studies because of its convenience and safety for clinical application ${ }^{17}$. However, varying protocols of limb RIPoC were selected in different studies, 
while the optimal one remains to be determined. In the present study, two experimental protocols of limb RIPoC were evaluated. Limb RIPoC1 applied as 3 cycles of 5-minute ischemia/5minute reperfusion has been proven as a valid protection against $\mathrm{I} / \mathrm{R}$ injury in brain ${ }^{8,19}$, while Limb RIPoC2 with 1 cycle of 15-minute ischemia has been proven to confer a strong degree of cardioprotection against I/R injury ${ }^{20}$. Our findings indicated that limb RIPoC1 significantly reduced the flap necrosis area at the $7^{\mathrm{h}}$ days after reperfusion compared to the controls. On the contrary, limb Rchemiadid not provide equihemia protection for the flap.

The optimal number of stimuli cycles applied in RIPoC remains a controversial subject. Some researchers concluded hat pProtection by remote conditioning requires the completion of a sufficient stimulus during index ischemia. Study by Kanoria et $a l{ }^{21}$ demonstrated that remote conditioning with multiple cycles of $\mathrm{I} / \mathrm{R}$ stimuli was more effective than with a single cycle of stimulus in cardioprotection. Loukogeorgakis et al..$^{22}$ reported that $\mathrm{I} / \mathrm{R}$ injury was significantly prevented by an arm RIPoC with 3 cycles of 5-minute ischemia/5-minute reperfusion, but shortening the stimulus to 2 cycles was much less protective, indicating that $\mathrm{RIPoC}$ is a dose-response protection. In the present study, in spite of the same total duration of limb ischemia, RIPoC1 rather than RIPoC2 was found effective in limiting flap I/R damage, which supports the hypothesis that multiple cycles of short limb RIPoC was more effective than a single cycle of long limb RIPoC.

Understanding the mechanisms responsible for the ischemic conditioning therapy is important for its clinical practice which aims at mimicking the protective mechanisms. In agreement with previous studies ${ }^{23,24}$, high level of oxidative stress was induced in the rat flap subjected to 8 -h ischemia, demonstrated by increased MDA contents and decreased SOD activities. The level of MDA indirectly reflects the severity of cellular injury from free radical attack, while SOD activity indirectly reflects the level of scavenging capacity. When the oxidative stress occurred, the rapid overproduction of free radicals overwhelms the cellular detoxification and scavenging capacity within the body ${ }^{25}$. In the present study, however, these changes were attenuated by limb RIPoC1 conducted immediately after reperfusion in the flap. Compared to the Control group, limb RIPoC1 produced an increased MDA contents appearing at $6 \mathrm{~h}$ after reperfusion, and a decreased SOD activities appearing at $12 \mathrm{~h}$ after reperfusion, while the differences in these parameters between the Control and RIPoC2 groups were insignificant. Consistent with the previous studies that demonstrated the antioxidative effect of RIPoC on liver and kidney ${ }^{26}$, our results provide promising evidence for its effect on decreasing the production of peroxidation products and increasing free radical scavenging capacity in the early stage of flap reperfusion injury.

There's limitation that should be mentioned. Since flap necrosis was the primary endpoint, based on our experience and the previous literature ${ }^{23}, 8$-hour ischemia was conducted to establish a stable model of $\mathrm{I} / \mathrm{R}$ injury with partial flap loss. Therefore, our findings might focus on flaps with severe ischemia induced by vaso-occlusive crisis, when RIPoC could be applied as an add-on therapy to re-explorations. Its effect on routine free flap transplantation, in which warm ischemia time usually does not exceed 2 hours, was nevertheless not well discussed in the current study. Thus, further studies based on a model with different ischemia time are needed, and assessments of acute biomarkers of $\mathrm{I} / \mathrm{R}$ such as neutrophil infiltration are recoended.

\section{Conclusions}

The remote ischemic post-co thatRIPoC applied as 3 cycles of 5-min limb ischemia followed by 5-min reperfusion limits $\mathrm{I} / \mathrm{R}$ injury ineprotective and IPoC may beis mediated by limiting oxidatap tissues in the early stage of reon. Thus, ilntervention with RIPoC, which targets the first few minutes of reperfusion, may be a valuable clinical “after-injury strategy' for free flap research.

\section{References}

1. Siemionow, Arslan E.. Ischemia/reperfusion injury: a review in relation to free tissue transfers. Microsurgery. 200424(6):468-475. PMID: 15378577.

2. Sun L, Li Y, Shi J, Wan X, Wang X:. Protective effects of ligustrazine on ischemia-reperfusion injury in rat kidneys. Microsurgery. 20022(8):343-346. PMID: 12497570.

3. Im MJ, Manson PN, Bulkley GB Hoopes JE:. Effects of superoxide dismutase and allopurinol on the survival of acute island skin flaps. Ann Surg. 1985;1(3):357-359. PMID: 3977440.

4. Wang KX, Hu SY, Jiang XS, Zhu M, Jin B, ZhangGY, Chen B: Protective effects of ischaemic postconditioning on warm/cold ischaemic reperfusion injury in rat liver: a comparative study with ischaemic preconditioning. Chin Med J (Engl). 2008;120):20042009. PMID: 19080265.

5. Ren C, Gao X, Niu G, Yan Z, Che X, Zhao H:. Delayed postconditioning protects against focal ischemic brain injury in rats. PLoS One. 2008;3(12):e3851. PMID: 19066627.

6. Ma XJ, Zhang XH, L CM, Luo M:. Effect of postconditioning on coronary blood flow velocity and endothelial function in patients with acute myocardial infarction. Scand Cardiovasc J. 200640(6):327-333. PMID: 17118822.

7. Li S, Wu J, Watanabe M, Li , Okada T.: Protective effects of ischemic postconditioning against hypoxia-reoxygenation injury and hydrogen peroxide-induced damage in isolated rat hearts. Exp Clin Cardiol. 20061(4):280-285. PMID: 18651018.

8. Li CM, Zhang XH, M XJ, Luo M.. Limb ischemic postconditioning protects myocardium from ischemia-reperfusion injury. Scand Cardiovasc J. 20060(5):312-317. PMID: 17012143. 
9. Hess DC, Hoda M, Bhatia K:. Remote limb perconditioning [corrected] and postconditioning: will it translate into a promising treatment for acute stroke? Stroke. 2013;4):1191-1197. PMID: 23339961.

10. Grall S, Prunier-Mirebeau D, Tamareille S, Mateus V, Lamon D, Furber A Prunier F.: Endoplasmic reticulum stress pathway involvement in local and remote myocardial ischemic conditioning. Shock. 20139(5):433-439. PMID: 23481493.

11. Moon JG, Lim HC, Gye MR, Oh S, Park JW:. Postconditioning attenuates ischemia-reperfusion injury in rat skin flap. Microsurgery. 20088(7):531-537. PMID: 18683865.

12. Coskunfirat OK, Cinpolat A, Bektas G, OganO, Taner T. Comparing different postconditioning cycles after ischemia reperfusion injury in the rat skin flap. Ann Plast Surg. 20142(1):104-107. PMID: 23503428 .

13. Kerendi F, Kin H, Halkos ME, Jiang R, Zatta AJ, Zhao ZQ, Guyton RA, VintenJohansen J:. Remote postconditioning. Brief renal ischemia and reperfusion applied before coronary artery reperfusion reduces myocardial infarct size via endogenous activation of adenosine receptors. Basic Res Cardiol. 2005;00(5):404-412. PMID: 15965583.

14. Ren C, Yan Z, Wei D, Gao X, Che X, Zhao H.. Limb remote ischemic postconditioning protects against focal ischemia in rats. Brain Res. 2009;1288:88-94. PMID: 19631625.

15. Lee JI, Wook Nha K, Suh JS, Choo SK, Park H, Park JW:. Remote postconditioning attenuates ischemia/reperfusion injury in rat skeletal muscle through mitochondrial ATP-sensitive $\mathrm{K}+$ channeldependent mechanism. J Reconstr Microsurg. 20139(9):571-578. PMID: 23757151.

16. Crimi G, Ferlini M, Gallo F, Sormani MP, Raineri C, Bramucci E, De Ferrari GM, Pica S, Marinoni B, Repetto A, Raisaro A, Leonardi S, Rubartelli P, Visconti LO,Ferrario M:. Remote ischemic postconditioning as a strategy to reduce acute kidney injury during primary PCI: a post-hoc analysis of a randomized trial. Int J Cardiol. 2014;7(2):500-502. PMID: 25183541.

17. Vinten-Johansn J, Shi W:. The science and clinical translation of remote postconditioning. J Cardiovasc Med (Hagerstown). 201314(3):206-213. PMID: 23412366.

18. Sun J, Tong L, Luan Q, Deng J, Li Y, Li Z, DongH, Xiong L: Protective effect of delayed remote limb ischemic postconditioning: role of mitochondrial K(ATP) channels in a rat model of focal cerebral ischemic reperfusion injury. J Cereb Blood Flow Metab. 20122(5):851-859. PMID: 22274742.

19. Hu S, Dong H, Zhang H, Wang S, Hou L, Chen S, ZhangJ, Xiong L:. Noninvasive limb remote ischemic preconditioning contributes neuroprotective effects via activation of adenosine A1 receptor and redox status after transient focal cerebral ischemia in rats. Brain Res. 2012;1459:81-90. PMID: 22560096.

20. Basalay M, Barsukevich V, Mastitskaya S, Mrochek A, Pernow J, Sjoquist PO, Ackland GL, Gourine AV Gourine A:. Remote ischaemic pre- and delayed postconditioning - similar degree of cardioprotection but distinct mechanisms. Exp Physiol 201297(8):908-917. PMID: 22427438.

21. Kanoria S, Jalan R, Seifalian AM, Williams R, avidson BR:. Protocols and mechanisms for remote ischemic preconditioning: a novel method for reducing ischemia reperfusion injury. Transplantation. 20074(4):445-458. PMID: 17713425.

22. Loukogeorgakis SP, Williams R, Panagiotidou AT, Kolvekar SK, Donald A, Cole TJ, Yellon DM, Deanfield JE, Macllister RJ: Transient limb ischemia induces remote preconditioning and remote postconditioning in humans by a K(ATP)-channel dependent mechanism. Circulation. 2007;1112):1386-1395. PMID: 17724264.
23. Aslan C, Melikoglu C, Ocal I, Saglam G, Sutcu R, osnuter M. Effect of epigallocatechin gallate on ischemia-reperfusion injury: an experimental study in a rat epigastric island flap. Int J Clin Exp Med. 2014;7(1):57-66. PMID: 24482689.

24. Celik A, Ersoy OF, Ozkan N, Kayaoglu HA, Ozugurlu F, Cakir EA, Lordlar N,Omeroglu S:. Comparison of the effects of troxerutin and heparinoid on flap necrosis. J Plast Reconstr Aesthet Surg. 201063(5):875-883. PMID: 19346174.

25. Loeper J, Goy J, Klein JM, Dufour M, Bedu O, Loeper, Emerit $\mathrm{J}$ :. The evolution of oxidative stress indicators in the course of myocardial ischemia. Free Radic Res Commun. 1991;12-1 Pt 2:675680. PMID: 2060839.

26. Costa FL, Teixeira RK, Yamaki VN, Valente AL, Silva AM, Brito MV,Percario S:. Remote ischemic conditioning temporarily improves antioxidant defense. J Surg Res. 2016 Jan;200(1):105-9. doi: 10.1016/j.jss.2D:26316445

\section{Correspondence:}

Jiasheng Dong M.D.

Department of Plastic and Reconstructive Surgery

Shanghai Ninth People's Hospital

Shanghai Jiao Tong University - School of Medicine

No. 639 Zhizhaoju Road Shanghai (200011) - China

Phone: 008613701654987

dongjiasheng_9y@163.com

Received: Sept 22, 2015

Review: Nov 17, 2015

Accepted: Dec 19, 2015

Conflict of interest: none

Financial source: none

${ }^{1}$ Research performed at Shanghai Ninth People's Hospital, School of Medicine, Jiao Tong University, China. 